\title{
Oscillations in solar Jets Observed with the SOT of Hinode: \\ Viscous Effects during Reconnection
}

\author{
E. Tavabi ${ }^{1}$ and S. Koutchmy ${ }^{2}$ \\ 1-Physics Department, Payame Noor University, 19395-4697 Tehran, I.R. of Iran \\ 2- Institut d'Astrophysique de Paris (CNRS) \& UPMC, UMR 7095, 98 Bis Boulevard Arago, F-75014 Paris, \\ France \\ (E-mail: tavabi@iap.fr and koutchmy@iap.fr).
}

\begin{abstract}
.
Transverse oscillatory motions and recurrence behavior in the chromospheric jets observed by Hinode/SOT are studied. A comparison is considered with the behavior that was noticed in coronal X-ray jets observed by Hinode/XRT. A jet like bundle observed at the limb in Ca II H line appears to show a magnetic topology that is similar to X-ray jets (i.e., the Eiffel tower shape). The appearance of such magnetic topology is usually assumed to be caused by magnetic reconnection near a null point. Transverse motions of the jet axis are recorded but no clear evidence of twist is appearing from the highly processed movie.

The aim is to investigate the dynamical behavior of an incompressible magnetic X-point occurring during the magnetic reconnection in the jet formation region. The viscous effect is specially considered in the closed line-tied magnetic X-shape nulls. We perform the MHD numerical simulation in 2-D by solving the visco-resistive MHD equations with the tracing of velocity and magnetic field. A qualitative agreement with Hinode observations is found for the oscillatory and non-oscillatory behaviors of the observed solar jets in both the chromosphere and the corona. Our results suggest that the viscous effect contributes to the excitation of the magnetic reconnection by generating oscillations that we observed at least inside this $\mathrm{Ca}$ II $\mathrm{H}$ line cool solar jet bundle.
\end{abstract}

Key words. MHD oscillation- Sun: chromosphere- Sun: corona- reconnection jet.

\section{Introduction}

Magnetic reconnection is a dynamic process and among the most fascinating plasma processes that can be observed in the solar atmosphere over a wide range of wavelengths. Introductions to the magnetic reconnection processes in the solar corona can be found in textbooks (Priest \& Forbes 2000, Aschwanden 2009), or in recent review articles (e.g. Hood et al. 2002). Several recent studies deal with chromospheric reconnection processes that later contribute to coronal heating with propagating MHD waves near null points (location in the field where the magnetic field, and thus the Alfven speed, is zero) of the magnetic field (Sakai et al. 2002 and Shibata et al. 2007). Theoretical studies have been done in constructing analytic models of the oscillatory 
reconnection aspect on MHD waves following pioneering works (Rickard and Titov 1996; McLaughlin and Hood 2005 and McLaughlin et al. 2009). More recently, sophisticated numerical models of reconnections in the corona appeared (Pariat et al. 2010), fitting what is observed using XRT (Savcheva et al. 2007 and 2009); they include recurrent phenomena. Let us however note that a topological reconnection occurs only in a non collisional plasma which is not the case in the inner corona where, in addition to being collisional, the plasma radiates and possibly, even the solar gravity could play some role (further we will neglect these effects).

Magnetic reconnection process in the solar atmosphere may generate quasi-periodic oscillations with non-regular periods of the order of 2-10 minutes and damping times of several tens of minutes in radio, optical, and X-EUV emissions in loops of flaring regions (Kliem et al. 2000; Ofman and Sui 2006; Inglis and Nakariakov 2009) and active regions (De Moortel et al, 2000), which are the most likely evidence of reconnection generating propagating magnetoacoustic waves. Outside of active regions coronal oscillations were reported off- disk in the range of 5 min periods (Tsubaki, 1977; Tomczyk et al. 2007) and also at shorter periods (Koutchmy et al. 1983). Evidences of such dynamical processes were also reported for eruptive filaments (e.g., Bocchialini et al. 2011), and magnetoacoustic waves were recorded on- disk inside an anomalously bright network rosettes using simultaneous observations in 3 photospheric and chromospheric lines (Baudin et al., 1996). When considering a higher spatial resolution, even finer scales and faster variations are observed (Cirtain et al. 2013).

In spite of reconnection generating wave phenomena, such magnetic field rearrangements and related processes may generate also various types of solar transients such as jets, spicules, mottles, fibrils etc. at different spatio-temporal scales of the solar atmosphere. The solar X-ray jets can be triggered by dynamical reconnection processes (e.g., Yokoyama and Shibata, 1995, 1996; Nishizuka et al., 2008; Culhane et al. 2007; Filippov et al., 2012, Filippov et al., 2009, Pariat et al. 2010). Overall, magnetic reconnection processes are an important candidate to launch solar jets and induce oscillatory phenomena in the solar atmosphere.

Ofman et al. (1991) investigated the visco-resistive MHD equations and found that the beams close to current layers can lead to the onset of Kelvin-Helmholtz instability and to overstable (i.e. oscillatory) modes. Hasam (1992) and Ofman et al. (1993 and 1994) have also investigated the normal mode solutions for $\mathrm{m}=0$ and $m \neq 0$ modes with resistivity and viscous effect included. Craig et al. (2005) showed that viscosity can indeed have a profound effect on the dynamics of magnetic reconnection. They point out that viscosity can dissipate not only the kinetic energy but also a significant fraction of magnetic energy. Accordingly, the free magnetic energy can be dissipated by oscillatory reconnection. Such effects may have significant implications in driving the jets in the solar atmosphere. Oscillatory reconnections were studied in several papers that do show a clear evidence for propagating waves in jets (Cirtain et al. 2007, He et al. 2009 and Morton et al. 2012). They found that a series of reconnection reversals (or oscillatory behavior) take place; during each burst the gas pressure gradient in the reconnection site must be balanced by the Lorentz force generated by the combined action of the magnetic 
pressure gradient acting toward the current sheet and the magnetic tension force pulling the curved field lines away from the current sheet.

In the present paper, we first report the observations of a chromospheric bundle of jets and also consider similar in morphology X-ray jets for comparison, including those studied in the litterature. We then numerically model the role of viscous effects in the reconnection induced oscillatory and non-oscillatory processes near the incompressible magnetic X-point, and qualitatively compare those results with the observed solar jets.

\section{Hinode Observations from the SOT.}

\section{2-1 Data and Image processing.}

The Solar Optical Telescope (SOT) onboard Hinode addresses many questions of solar magneto-hydrodynamics. Data taken above the solar limb can be used to study the dynamical cool jets, prominences and surge like events. The CaII $\mathrm{H}$ line data used in this study are from the Broadband Filter Imager (BFI) of SOT. To a smaller extend we also used the H $\alpha$ data from the Narrowband Filter Imager (NFI) of SOT. Hot jets are studied using the XRT from the same spacecraft.

SOT very high resolution observations were performed sequentially during several minutes with different cadences near $20 \mathrm{sec}$, at the diffraction limit resolution of $\frac{\lambda}{D}$ or $0^{\prime \prime} .16(120 \mathrm{~km}) \mathrm{using}$ a $0^{\prime \prime} .054$ pixel size on filtergrams of the $\mathrm{Ca}$ II $\mathrm{H}$ line and roughly two times worse for $\mathrm{H} \alpha$ filtergrams (Tsuneta et al. 2008): the spatial resolution with the diffraction limit is now $0^{\prime \prime} .33$ $(250 \mathrm{~km})$ and the scale is $0^{\prime \prime} .08 /$ pixel (see Figure 1 ). 


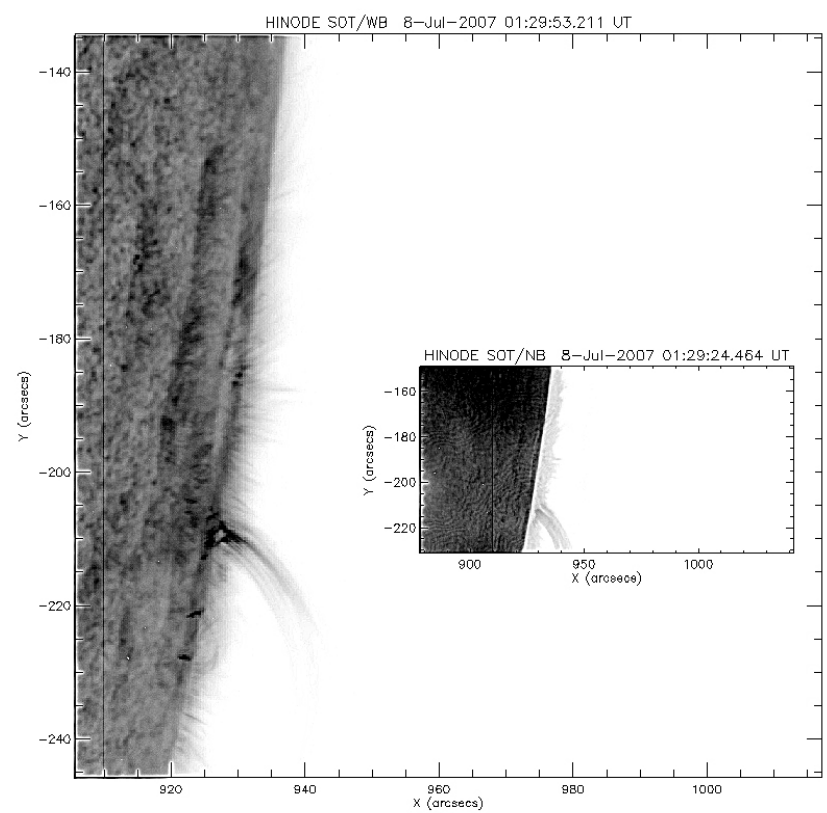

Figure 1. Negative and processed $\mathrm{Ca}$ II $\mathrm{H}$ and simultaneous $\mathrm{H} \alpha$ images showing the studied cool jet; the simultaneously obtained $\mathrm{H} \alpha$ image is shown inserted in the $\mathrm{Ca}$ line image; times are shown in UT.

The size of all images which were used is $1024 \times 1024$ pixels $^{2}$ (Hinode read out only the central pixels of the detector to keep the high cadence in the telemetry restrictions) thus covering an area of (FOV) $111^{\prime \prime} \times 111^{\prime \prime}$. The Hinode SOT data were calibrated with the standard 'fg_prep' of the SSW software (Shimizu et al. 2008). A superior spatial image processing for thin thread-like and aligned features is obtained using the mad-max algorithm (Koutchmy \& Koutchmy 1989). The mad-max operator substantially enhances the finest scale structure. It is a weakly nonlinear modification of a second derivative spatial filter. Specifically, it is where the second derivative has a maximum when looking along different directions (usually, 8 directions). The behavior of mad-max qualitatively resembles the second derivative, but the strong selection along the direction of the maximum variation substantially enhances thread-like structures. It also appears to reduce the blending between crossing threads caused by the superposition effect along the line of sight. The algorithm, as originally proposed, samples the second derivative in eight directions, but the directional variation of the second derivative was generalized to a smooth function with a selectable pass band spatial scale for this work (for more details see November \& Koutchmy 1996). Spatial filtering using "mad-max" algorithms clearly shows fairly bright radial threads in the chromosphere at a scale as fine as the resolution limit of about $120 \mathrm{~km}$ (Tavabi and Koutchmy, 2012). 


\section{2-2 Event Description}

\section{2-2-1 SOT (Hinode) Ca II H line observation}

Many small transient brightenings were observed (see the accompanying movie) inside the mini- loop system of Fig. 1 for about two-three hours on 2007 July 08, between about 00:34 and 03:11 UT.

Figure 2 shows consecutive processed snapshots outlining the main feature of the jet in $\mathrm{Ca}$ II $\mathrm{H}$ line, starting at 01:28 UT, when the jet spine part becomes visible. The red dashed lines denotes the average axis of the jet (taking into account the occurrence of several components) and the short blue line across shows the height where transverse displacements are further analyzed. We see no evidence of crossings between the different components of the jet, clockward or anticlockward, which means no sign of twist taking into account the 3D distribution. The brightenings occurring near the feet of the system will not be analyzed here.

At about 01:25 UT, the jet becomes more dynamical and several null points (evidence of inverse Y shaped parts) appear close to each other. The whole system appears to have changing intensities and in overall, possibly show a small scale anemone jet-like structure as defined by Shibata et al. 2007 for X-ray jets, or an Eiffel tower shape as defined by others, starting with Zirin and Cameron 1998.

This observation also suggests that the dynamical phenomena at feet could be caused by the interaction of newly emerging small magnetic flux in the arcade like field near some active region but this is just speculations because no magnetic field measurements exist in this region and we could not find this region on the Stereo A spacecraft filtergrams when looking at the Wlimb region (the spacecraft was situated at 12 degrees before the Earth looking at the Sun). The jet-like event is evaluated as a faint brightening propagating along the spine; a slingshot behavior is clearly seen from Fig. 7; it could be understood assuming some model of magnetic reconnections between small emerging dipoles. This whip-like motion with brightenings also include a small amplitude transverse oscillation which maybe related to a magnetoacoustic wave similar to a propagating kink or an Alfvenic wave, although no evidence of twist is detected. Let us add that this event is a really small scale event although significantly taller than the typical spicules confined to the 4 to $6 \mathrm{Mm}$ heights. Accordingly, this event could barely be identified on 304 available filtergrams from EIT (SoHO) or from the higher quality Stereo filtergrams taken at the same time, which is a pity because the Stereo spacecrafts were ideally situated for a 3D viewing. On the noisier at these scales coronal filtergrams, nothing could be found. 


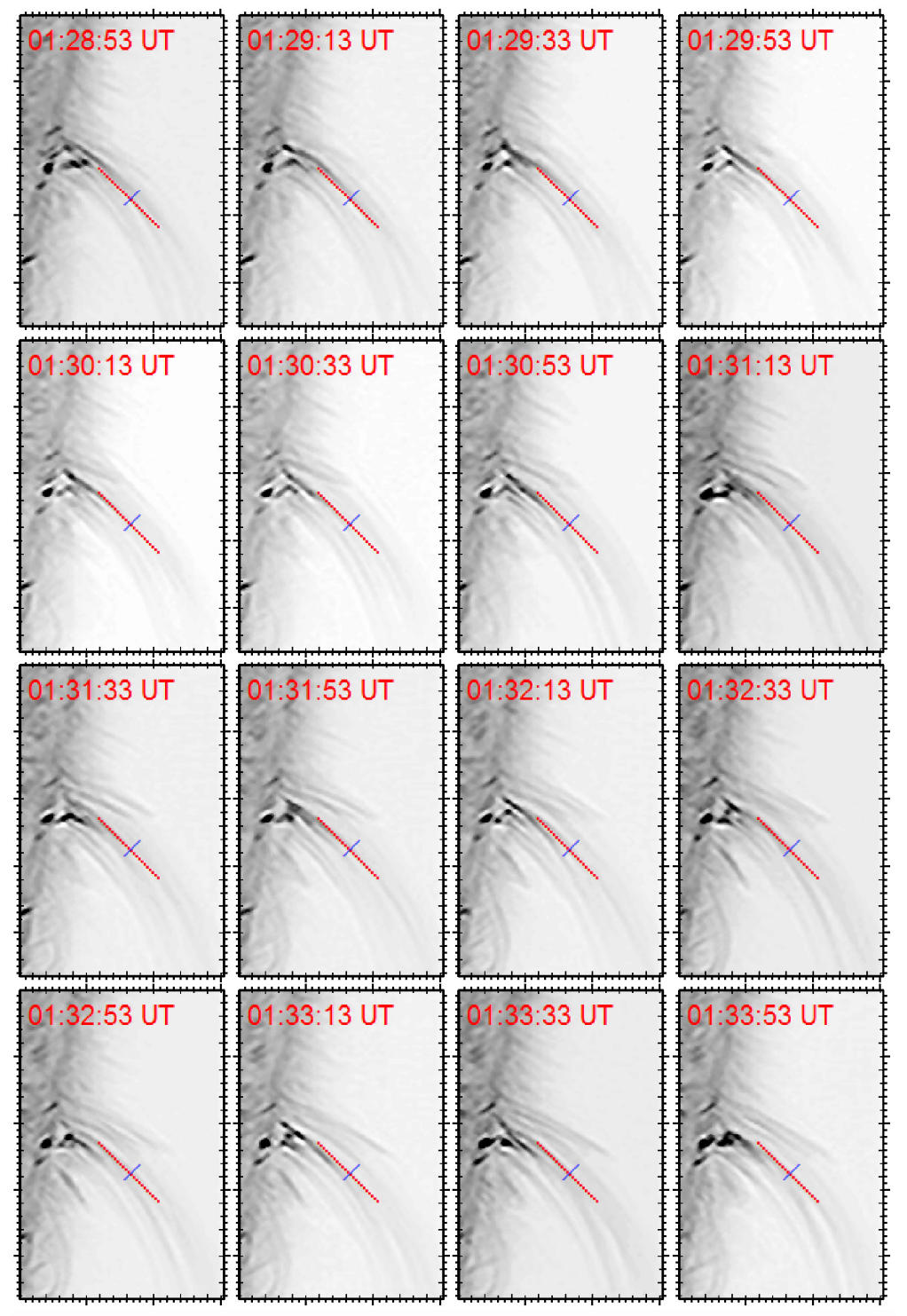

Figure 2. Selected snapshots of negative and mad-maxed images from the SOT (Hinode) broadband Ca II H filter observations $\left(15 \times 25 \operatorname{arcsec}^{2} \mathrm{FOV}\right)$ at different times (01:28 to 01:33 UT). Tick marks show .5 arcsec. The spine axis (taking into account all components) are denoted by a dashed red line. The blue line shown in all images and well above the reconnection site position, outlines the location where oscillatory transverse motions and displacement of the most central part are observed, see results in Fig. 7.

A first data analysis of this CaII event was described in a preliminary paper (Tavabi et al. 2011) where we concentrated on the oscillatory behavior of the CaII jet by using a wavelet analysis performed at 2 different heights. By analyzing the correlations we deduced a phase velocity of 
upwards propagation at selected frequencies. From the analysis of the power around the 5.5 $\mathrm{mHz}$ frequencies we deduced a phase velocity of 30 to $40 \mathrm{~km} / \mathrm{s}$ corresponding to a measured 1 min time delay between the 2 heights. We do not duplicate here this analysis (Tavabi et al. 2011) and proceed to discuss some new more general results relevant to the jets physics.

\section{2-2-2 XRT (Hinode) observation.}

XRT is a high-resolution grazing-incidence telescope, which provides unprecedented highresolution and high cadence observations of the X-ray corona through a wide range of filters (Golub et al. 2007). For the purpose of looking at the jet events similar in morphology to the preceding SOT event observed in CaII with an Eiffel tower shape, we selected the observation shown on Fig. 3 following our experience in studying XRT jets (Filippov et al. 2009, 2012).

We use here the filter combination Ti-poly/Open. The exposure time was $8 \mathrm{~s}$ or $16 \mathrm{~s}$, the cadence was about 53 s. These Hinode XRT data were also calibrated with the standard 'xrt_prep' software (Shimizu et al. 2008). In Fig. 3 we show such typical jet in hot coronal line emission (soft x-ray jet). The total evolution time is shorter than for the preceding case of a SOT cool jet (less than 12 min.). An extended analysis of XRT jets was already presented (Savcheva et al. 2007, 2009) but we should notice that the achieved spatial resolution is not as good as in the case of SOT CaII observations, not only because the pixel size is much smaller for SOT observations, but also because the amount of available photons is larger, which makes the $\mathrm{S} / \mathrm{N}$ ratio higher and the exposure time shorter to reduce the smearing effect of jittering. 


\section{Hinode/XRT 2009-March-14}

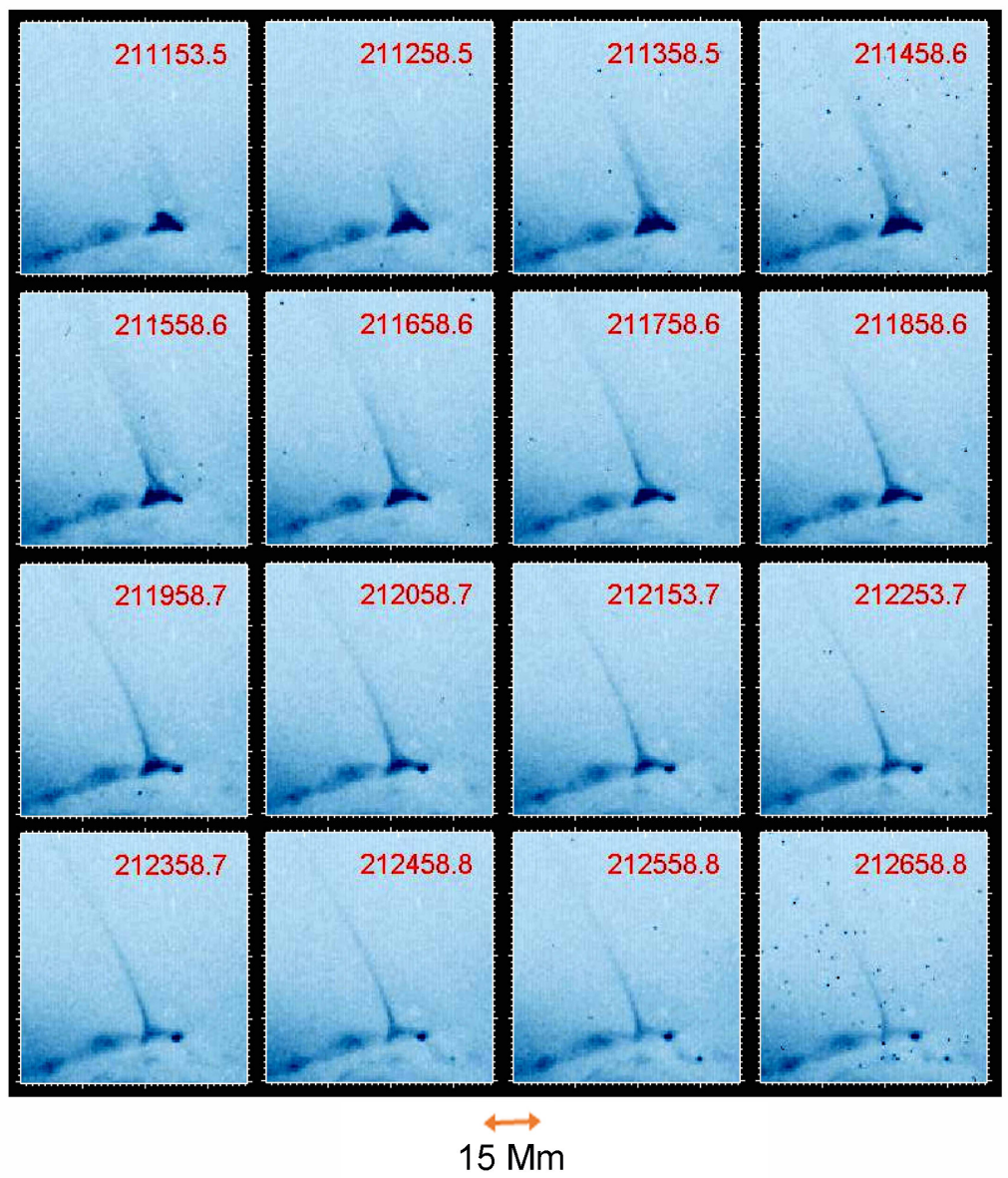

Figure 3. Negative $X$-ray images processed with madmax. They are extracted from a sequence of XRT (Hinode) observations taken on 14 March 2009 showing a typical Eiffel tower type jet in hot coronal X-ray lines. It illustrates the similarity of structures recorded with both the XRT and the SOT in CaII H (see Fig. 2) assuming that near 21:15 several components are indeed recorded but they are not fully resolved due to the limited resolution.

The event (Fig. 3) occurs at the north coronal hole region, near the photospheric limb, with an excellent contrast. We found the structure of this hot X-ray jet is similar to what is observed in the preceding cool jet, although this event is of larger scale and occurs at higher latitudes. The typical width of the feet is now of order of 20 " or $14 \mathrm{Mm}$ (see figure 3) compared to 3" to 4" for the cool jet of figure 2. The occurrence of several brightening at the feet of the event and the Eiffel tower structure of the jet, with a transverse motion of the spine, are signatures that support the comparison, provided we imply a general magnetic origin with reconnexions. Short faint brightenings in SXR occurring in polar regions were indeed already described from Yohkoh “deep" SXT observations (Koutchmy et al. 1997,1998), where they used the terminology of 
"flashes" under their SXR polar jets at time of solar minimum (1997). For the much brighter Xray jets related to active regions, we remind that the corresponding SXR magnetic structure was discovered in the SXT images of Yohkoh and called "anemone" in the original paper of Shibata et al. 1997, suggesting they are made of loops. Similar structures were episodically observed on W-L good eclipse pictures for a long time (Koutchmy, 1969; Waldmeier, 1972; Koutchmy et al. 1994 etc.). Interpretation of cool jet structures in term of wave was also discussed (Erdelyi and Fedun, 2007).

Because the SOT observations of Hinode taken in the CaII H line are today clearly of much better resolution then everything else obtained before, we now rather concentrate our attention to that case.

\section{Basic equations and numerical method}

We solve the time dependent viscosity and resistive MHD equations in a rectangular bounded two-dimensional domain (Gunzburger and Trenchea 2005). With these assumptions the basic dimensionless form of equations for a viscous incompressible resistive fluid is:

$$
\begin{aligned}
& \partial_{t} V+V . \nabla V-\nu \nabla^{2} V+\nabla P+S \nabla\left(\frac{1}{2} B^{2}\right)-S(B . \nabla) B=0 \\
& \partial_{t} B+(V . \nabla) B-(B . \nabla) V+\eta \nabla \times \nabla \times B=0
\end{aligned}
$$

$V$ is the plasma velocity, $B$ the magnetic induction (usually called the magnetic field), $P$ is the plasma pressure all variables are normalized using the asymptotic Alfven velocity $V_{A}=\frac{B_{0}}{\left(\mu_{0} \rho_{0}\right)^{\frac{1}{2}}}$ of the configuration at $\mathrm{t}=0$ and $\mu_{0}=4 \pi \times 10^{-7} \mathrm{Hm}^{-1}$ is the magnetic permeability, time is measured in units of the Alfven time $\tau_{A}=\frac{l}{V_{A}}$ and $l$ is the typical thickness of the current layer half-width. $\quad \eta$ is the magnetic diffusivity normalized by $\mu_{0} l V_{A}$, then the magnetic Reynolds number is given by $R_{m}=\eta^{-1}$ (it is a measure of the strength of the coupling between the flow and the magnetic field) and the Reynolds number $R_{e}=v^{-1}$ (where $V$ is the scalar kinetic viscosity), $S=\frac{H a}{R_{e} R_{m}}$ (where Ha is the Hartman number representing the ratio of magnetic to viscous diffusion forces), we take the equilibrium density to be uniform, i.e. $\rho=\rho_{0}$. Note that the scalar classical viscous term used here is not the most proper one for the solar corona; the viscosity should be a non-isotropic tensor.

A uniform grid in $\mathrm{x}, \mathrm{y}$ is used and the convergence is checked by looking at normalized differences in successive iteration in a domain with $(128)^{2}$ and the Eulerian backward approximation is used for solving the magnetic incompressible fluid flow problem into a rectangular domain. $\nabla . \mathrm{B}$ is initially zero; it is maintained at zero with the computer precision 
throughout the evolution. There are two specified parabolic inflows at the bottom part of the left and right boundary and outflows at lower and upper boundary where $\nabla \mathrm{V} . n=0$ and otherwise it is zero. The finite element techniques with piecewise linear function on triangles for the velocity (Taylor-Hood element) and quadratics were used for the magnetic field. Our magnetic field structure has a negative polarity embedded in a uniform positive one which corresponds to a $\{+$ $-+\}$ region. During the first phase of emergence, two flux tubes arise toward a non-magnetized medium and the size of the tubes increases. The initial state consists of a hydrostatic atmosphere with two magnetic flux loops; variables are made dimensionless. The interaction between the two magnetic loops leads to formatting a reconnection jet. The Jet was produced by forcing the interaction of the flux tubes changing the lateral distance between them. This simulation is performed by solving the 2-D dimensional viscosity and resistive MHD equations without gravity force, no thermal conduction and no radiative cooling effects.

\section{Results and Discussion}

In this section, we describe our numerical results and compare them with the XRT and especially the SOT CaII H line observations. Fig. 4 shows the field line topology (in green) at a stage when the X-null point forms, and the background color of absolute value of velocity are shown in red.

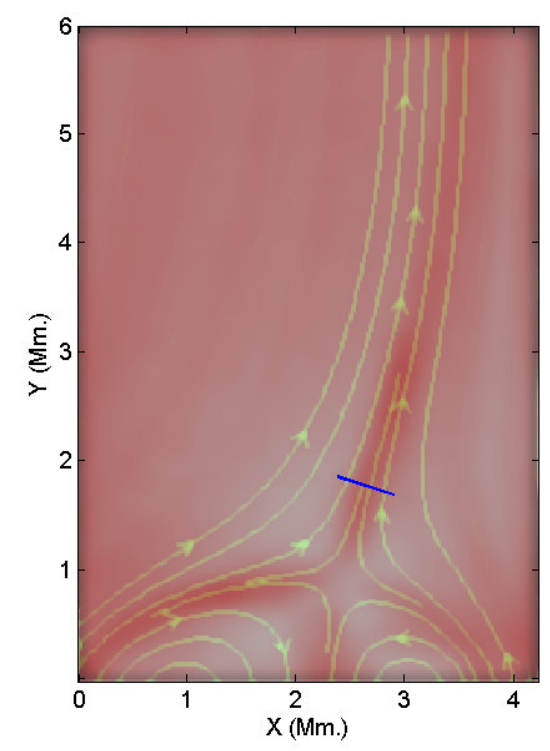

Figure 4. The magnetic field lines (in green) near the reconnection site and the absolute value of velocity $|V|$ are in red. The blue line is where we look at oscillations, see also Fig. 2.

The transverse motion computed above the reconnection site and along the short oblique blue line of figure 4 is shown as time-slice diagrams in Fig. 5. 
The oscillation and the decay disturbance of the total energy (magnetic and kinetic) for the cases corresponding to Fig.5 are respectively plotted in Fig. 6.
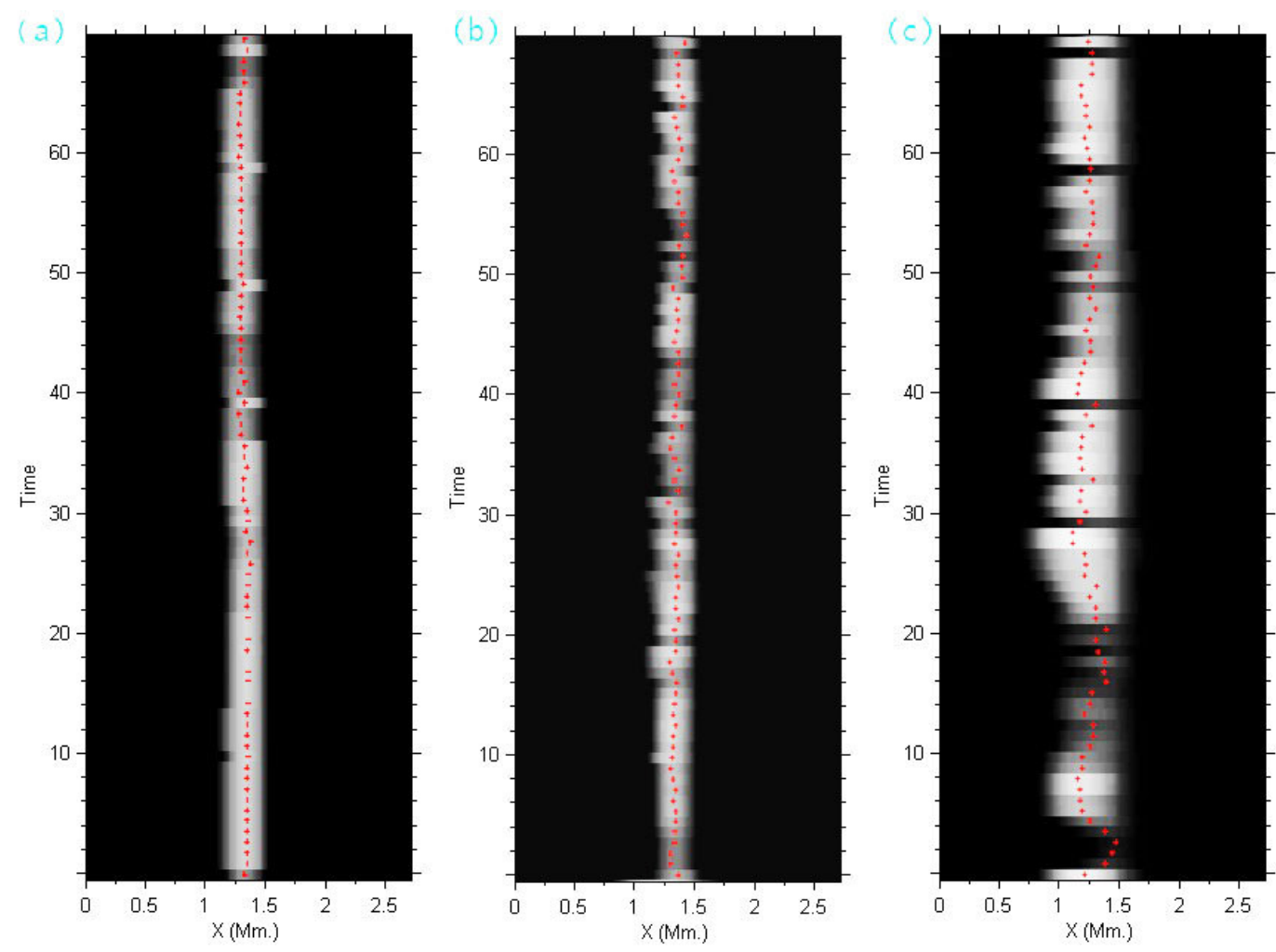

Figure 5. The quasi-periodic oscillatory behavior given by the viscosity effects. Left panel (Fig. 5-a) shows the behavior of the jet axis perturbation when $R_{e}=10^{3}$ and $R_{m}=10^{4}$ (or $v=0.001$ and $\eta=0.0001)$. The red crosses show the axis of jet. When the resistivity is raised $\left(R_{e}=R_{m}=10^{3}\right)$ the oscillatory aspect appears (fig. 5-b) and it is clearly seen in the right panel (fig. 5-c) for $R_{e}=10^{3}$ and $R_{m}=10$, the vertical axes are time in Alfvenic time-scale $T\left(\tau_{A}\right)$.

The results show that the integrated energy variations of the MHD fluid depend of the ratio of the Reynolds numbers; when this ratio $\frac{R_{m}}{R_{e}}$ is large, we see a rapid decay in the integrated energy (fig. 6 bottom panel), and by raising this ratio a typical oscillation will occur and the decay time is slower (fig. 6- top and middle panels). 

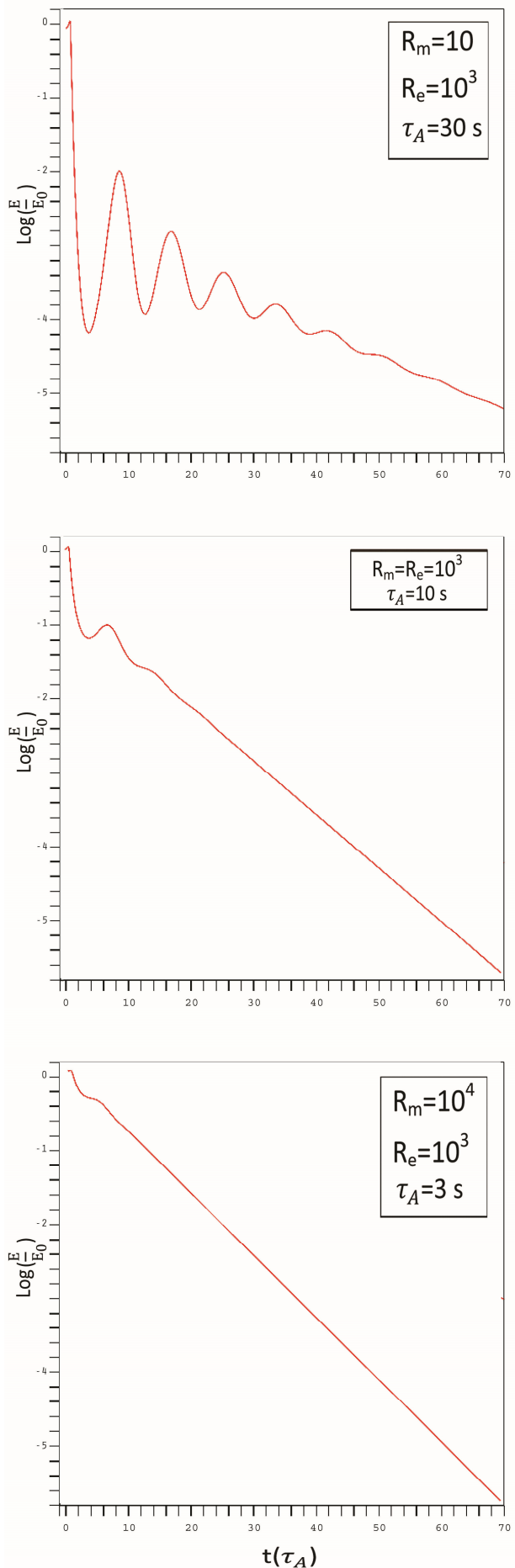

Figure 6. Energy fluctuations for different values of Reynolds numbers ratio. The vertical axis shows the value of the global energies $(E)$ for the initial equilibrium $\left(E_{0}\right)$; the horizontal axes are time in Alfvenic time-scale $T\left(\tau_{A}\right)$. 
Fig. 7 is a time/slice measured diagram which shows a temporal sequence of displacements corresponding to the axis of the jet (corresponding to the blue line in Fig. 2). With this diagnostic diagram we find that points on the jet spine axis oscillate transversally with time.

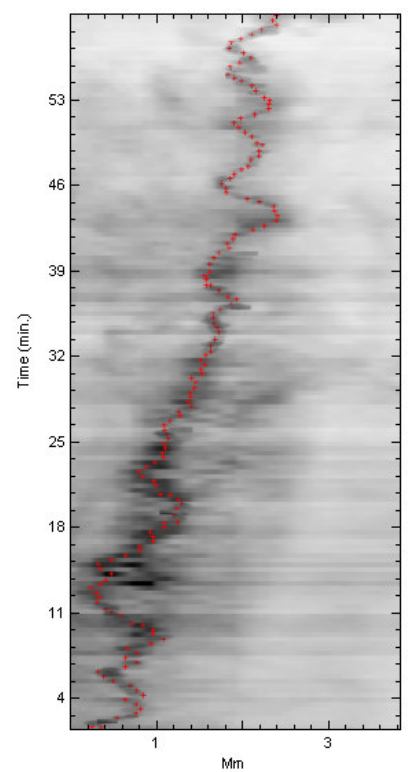

Figure 7- "Time slice" diagram using real images in taken in the "cool" Ca II line, using SOT/Hinode observations of figure 2 , to be compared to the time slice diagram of figure 5 .

\section{Discussion and conclusions}

We find evidence of oscillatory reconnections in both the adopted simulation and the presented Hinode/SOT CaII line (only a qualitative agreement is given) observations. For the atmospheric layers corresponding to two temperature regimes: $(i)$ the chromospheric structure evidenced in the cool Ca II H line and (ii) the XRT structure evidenced inside the coronal hole region, a good similarity seems to exist.

The X-ray jet of fig. 3 seems not to show any oscillation along the spine axis of the jet in intensity, taking into account the limited resolution; the spine is moving like a whip-like motion from left to right (see also Filippov et al. 2009), and seems to show a damping with a monotonic decay. This monotone damping is suggested with our simulation results for coronal conditions where the Reynolds numbers ratio is much greater than one (Fig. 6- bottom panel).

For the chromospheric dense and cool jet, the displacement of the ejected plasma flow is seen in fig. 7. This time-slice diagram shows quasi-periodic oscillations that are favorably predicted by 
the simulation result for $\frac{R_{m}}{R_{e}}<<1$ (fig. 6- top panel). For this case the "energy" damping shows a slow decay.

The azimuthally symmetric $(\mathrm{m}=0)$ modes are the only modes associated with topological reconnection and it is shown that reconnection can only occur in purely radial disturbances and allows a finite current parallel to the spine at the neutral point.

From the analysis by Rickard \& Titov (1996) it is clear that the $m=1$ mode (current accumulation in separator plane) is the most likely mode to occur for the axisymmetric single null point. There are two ways to generate this mode tilting and it results a current accumulation in the separator plane for a time interval that is shorter than he transient time of the driver, but for the double null point it is only possible to perturb each null with a pure $m=1$ mode by perturbing the spine axis.

From the theoretical interpretation point of view for the non-reconnected modes $(m>0)$ that include the scalar fluid viscosity, the fundamental oscillation period is $T\left(\tau_{A}\right) \approx \frac{1}{2}\left|\ln R_{m}\right|$ (Hasam 1992), and therefore, the Alfvenic time scale is $3 \mathrm{~s}$ for the corona and about ten times more for the chromospheric layers. And we know that the dissipation rate depends to the dissipative coefficient, thus for $R_{m}<R_{e}$ the problem is dominated by $\left|\ln R_{m}\right|$, and for $R_{e}<R_{m}$ it is dominated by $\left|\ln R_{e}\right|$.

It is clear that the viscous and the resistive terms can balance the Lorentz force and the advection, respectively. So the Lorentz force acting on the plasma can be diminished by viscosity and the global decay is restricted by the damping of the magnetic field energy on the resistive time scale and our results confirm this statement.

Rewriting the equation of motion as

$\rho \partial_{t} V=-\nabla P+J \times B+F_{v i s c}$

and neglecting the gravity, which is not relevant when we consider the horizontal pressure balance. The right hand side can be written as

$$
-\nabla\left(P+\frac{B^{2}}{8 \pi}\right)+\frac{1}{4 \pi}(B . \nabla) B+\rho \nu\left(\nabla^{2} V+\frac{1}{3} \nabla(\nabla . V)\right)
$$

When $R_{m} \gg R_{e}$ (or $\eta<<v$ ), the last terms of this equation is raising dramatically, so the balance between the tension force and the gradient of total pressure is falling down suddenly, and it leads to a fast burst outward of the diffusion region. This condition could be reversed when the viscosity coefficient is not large; then with $\eta \approx v$ it evidently provides a transition between the regimes of oscillation and of fast decay. The diffusion skin depth is defined as $\approx \eta^{\frac{1}{2}}$, then for $\eta>v$, after the first burst, the total pressure and tension force could be balanced again 
at the boundaries and the tension force will be marginally larger again, and the newly reconnected layer is reversed.

More simulation work is needed and more precise observational signatures should be considered, including densities and velocities. Unfortunately, the magnetic field cannot be directly measured. The new SDO mission data will help to develop and generalize such models, besides the SOT and the XRT Hinode data. The analysis of the short brightenings appearing at the feet of the Eiffel tower structure, part of loops or not, would need some more attention to understand the coronal "dynamics" of small emerging magnetic fluxes in these regions and their evolution above.

Acknowledgements. We thank B. Filippov for useful discussions. We are grateful to the Hinode teams for wonderful observations. Hinode is a Japanese mission developed and launched by ISAS/JAXA, with NAOJ as domestic partner and NASA and STFC (UK) as international partners. SOT is an experiment developed in cooperation with Lockheed Martin and XRT is an experiment developed in cooperation with the SAO of Harvard Univ. and NASA in cooperation with NAOJ. This work was supported by the

Center for International Scientific Studies \& Collaboration (CISSC) and by the French Embassy in Tehran in the frame of PHC.

\section{References}

Aschwanden, M. 2009, Physics of the solar corona, Springer, p 220

Baudin, F. Bocchialini, K. and Koutchmy, S. 1996, Astron. Astrophys. 314, L9

Bocchialini, K. Baudin, F. Koutchmy, S. Pouget, G. and Solomon, J. 2011, Astron. Astrophys. 533, 12

Craig, I. J., Litvinenko, Y. E. \& Senanayake, T., 2005, Astron. Astrophys. 433, 1139-1143

Cirtain, J. W., Golub, L., Lundquist, L., van Ballegooijen, A., Savcheva, A., Shimojo, M., DeLuca, E.,

Tsuneta, S. Sakao, T., Reeves, K., and 4 coauthors, 2007, Science, 318, 1580

Cirtain, J.W. Golub, L. Winebarger, A.R. et al. 2013, Nature, 493, 501

Culhane, L. Harra, L. K.; Baker, D., van Driel-Gesztelyi, L., Sun, Jian; Doschek, G. A., Brooks, D. H.,

Lundquist, L. L., Kamio, Suguru; Young, P. R., Hansteen, V. H., 2007, PASJ, 59, 751

DeMoortel, I. Ireland, J. and Walsh, R.W. 2000, Astron. Astrophys. 355, L23

Erdélyi,R. and Fedun, V. 2007, Science vol. 318, 5856, 1572

Filippov, B., Koutchmy, S. and Tavabi, E., 2012, Solar Phys. 286, 143

Filippov, B., Golub, L. and Koutchmy, S., 2009, Solar Phys., 254, 259

Golub, L. Deluca, E. et al. 2007, Solar Phys. 243, 63

Gunzburger,M. and Trenchea, C. 2005, J. Math. Anal. Appl. 308, 440-466

Hasam, A. B. 1992, ApJ, 399, 159

He, Jiansen; Marsch, Eckart; Tu, Chuanyi; Tian, Hui, 2009, ApJ, 705, 217

Hood, A. W., Brooks, S. J. \& Wright, A. N. 2002, Proc. Roy. Soc, A458, 2307

Inglis, A. R. \& Nakariakov, V. M., 2009, A\&A, 493, 259-266

Koutchmy, S. 1969, Astrophysical Letters, Vol. 4, 215 
Koutchmy, S. Zugzda, Y. D. and Locans, V. 1983, Astron. Astrophys. 120, 185.

Koutchmy, O. \& Koutchmy, S., 1989, in Proc. $10^{\text {th }}$ Sacramento Peak Summer Workshop, High

Spatial Resolution Solar Observations, ed. O. von der Luhe (Sunspot: NSO), 217

Koutchmy, S. Koutvitsky, V.A. Molodensky, M.M. Solov'iev, L.S. and Koutchmy, O. 1994,

Sp. Sc. Rev. 70, 1-2, 283

Koutchmy, S. Hara, H. Suematsu, Y. and Reardon, K. 1997, Astron. Astrophys. L. 320, L33

Koutchmy, S. Hara, H. Shibata, K. Suematsu, Y. and Reardon, K., 1998, in “Observational

Plasma Astrophysics: Five Years of Yohkoh and Beyond”, T. Watanabe et al. (eds.),

Kluwer Acad. Publ., 87-94.

Kliem, B., Karlicky, M., \& Benz, A. O., 2000, Astron. Astrophys., 360, 715

McLaughlin, J.A. \& Hood, A.W., 2005, Astron. Astrophys. 435, 313-325.

McLaughlin, J.A., De Moortel, I., Hood, A.W. \& Brady, C.S., 2009, Astron. Astrophys. 493, 227-240

Nishizuka, N., Shimizu, M., Nakamura, T., Otsuji, K., Okamoto, T. J., Katsukawa, Y.,

Shibata, K, 2008, ApJ, 683L, 83

November, L. J. \& Koutchmy, S. 1996, ApJ, 466, 512

Ofman, L. \& Sui, L., 2006, Astrophys. J., 644, L149

Ofman, L., Morrison, P. J. \& Steinolfson, R. S. 1993, Astrophys. J., 417, 748

Ofman, L., Davila, J. M. \& Steinolfson, R. S. 1994, Astrophys. J., 421, 360

Ofman, L., Morrison, P. J., Steinolfson, R. S., 1991, B.A.A.S., 23, 1042

Pariat, E. Antiochos, S. K. and DeVore, C.R. 2010, ApJ, 714, 1262

Priest, E. R., \& Forbes, T. G. 2000, Magnetic Reconnection: MHD Theory and Applications

(New York: Cambridge Univ. Press)

Rickard, G. J. \& Titov, V. S., 1996, Astrophys. J., 472, 840-852

Sakai, J. I., Nishi, K. and Sokolov, I. V., 2002, ApJ, 576, 1018

Savcheva, A. et al. 2007, PASJ, 59, 771

Savcheva, A. Cirtain, J.W. DeLuca, E.E. and Golub, L. 2009, ApJ. 702, L32

Shimizu, T., Nagata, S., Tsuneta, S., et al. 2008, Sol. Phys., 249, 221

Shibata, K. et al. (with 21 coauthors), 2007, Science, 318, 1591

Tavabi, E. Koutchmy, S. and Ajabshirizadeh, A. 2011, in 3rd School and workshop on Space

Plasma Physics, AIP Conf. Proc. 1356, 99- 105

Tavabi, E. and Koutchmy, S. 2012, Solar phys., 238, 187

Tavabi, E., Koutchmy, S., Ajabshirizadeh, A., 2012, Journal of Modern Physics, 3, 1786

Tavabi, E., Koutchmy, S., Ajabshirizadeh, A., 2011, Null-Point Oscillations of Chromospheric Large

Cool Jets, American Institute of Physics, 1356, 99.

Tomczyk, S. ; McIntosh, S.W. ; Keil, S.L. et al 2007, Science, 317, 1192

Tsubaki, T. 1977, Solar Phys. 51, 121

Tsuneta, S., Ichimoto, K., Katsukawa, Y., et al. 2008, Sol. Phys., 249, 167

Waldmeier, M. 1972, Solar Phys. 27, 143

Yokoyama, T. and Shibata, K., 1996, PASJ, 48, 353 
Yokoyama, T. and Shibata, K., 1995, Nature, 375, 42

Zirin, H., Cameron, R.: 1998, Dynamics of Solar Spicules, Solar Jets and Coronal Plumes, ESA, SP421, 39-41. 